\title{
Another approach to reliability measures for systems with correlated observations
}

\author{
W. Prószyński
}

Received: 29 October 2009 / Accepted: 4 June 2010 / Published online: 25 June 2010

(C) The Author(s) 2010. This article is published with open access at Springerlink.com

\begin{abstract}
An approach to internal reliability analysis is presented which, compared to existing approaches, offers further insight into the system responses to observation gross errors. The proposed reliability measures are defined on the diagonal and non-diagonal elements of the modified reliability matrix being an oblique projector. It maps the observations into the least squares residuals, both expressed as multiples of the a priori standard deviations of the observations. The system responses to a single gross error, such as the local, the quasi-global or the global response, are discussed and their consistency with non-correlation case is proved. Also, the reliability criteria interpretable in terms of those responses are proposed. They enable one to evaluate the observation systems with correlated and uncorrelated observations with respect to internal reliability. An attempt is also made to relate the global responses to reliability numbers that are the basis for determining the magnitude of the minimal detectable bias. Some differences of the new reliability measures in relation to existing measures are indicated. A numerical example is provided, showing the reliability analysis for a simulated horizontal network (distances, angles and GPS vectors), with given covariance matrix for GPS-vector components. In addition, a reliability analysis is presented for a simple leveling network being a study case for other authors referred to in this paper.
\end{abstract}

Keywords Oblique projector - Internal reliability . Disturbance/response relationship · Reliability measures . Redundancy numbers $\cdot$ Reliability numbers

W. Prószyński $(\varangle)$

Warsaw University of Technology, Pl. Politechniki 1,

00661 Warszawa, Poland

e-mail: wpr@gik.pw.edu.pl

\section{Introduction}

It was indicated in Wang and Chen (1994) that the redundancy numbers for correlated observations, unlike those for uncorrelated observations, may exceed the interval $\langle 0,1\rangle$ on both sides. It was concluded that internal reliability cannot be correctly measured by the redundancy number for correlated observations. Hence, a "generalized reliability number" having the range $\langle 0, \infty)$ was introduced, determined on the basis of the aforementioned redundancy number and the multiple correlation coefficient as an auxiliary parameter. In Schaffrin (1997) that measure was replaced by the "normalized reliability number", obtained by scaling it into $\langle 0,1)$ interval for improved interpretation and better comparability to redundancy numbers. However, the two approaches were reported (Schaffrin 1997) to yield a reverse ranking of measures for some specific cases. Still another attempt (Prószyński 1998) was to formulate reliability assessment for systems with correlated observations by extending the disturbance/response properties of the systems with uncorrelated observations. The comparative tests carried out for the three approaches failed to show a satisfactory solution. Additionally, it turned out that the above-mentioned reversing of ranks may occur, even for generalized reliability numbers being within the interval $\langle 0,1)$.

The contributions to reliability theory made by Teunissen $(1990,1996)$ were very helpful in further analyses. A general expression for reliability measure covering the case of correlated and uncorrelated observations made it possible to analyse the measures in terms of the minimal detectable bias (MDB), and provided a suitable geometric interpretation. It helped explain why the normalization procedure (Schaffrin 1997), developed to achieve better interpretation, may cause the reverse ranking of measures, and that neither of the two types of reliability numbers can signal the abnormal local responses of a network. 
This called into question the adequacy of one-dimensional description of network reliability and indicated the necessity of searching for another solution inclined towards twodimensional reliability measures.

Further research carried out by this author was focussed on the properties of oblique projectors that are asymmetric matrices. It was observed that there is some interrelation between each diagonal element and the degree of asymmetry in non-diagonal elements of the corresponding column and row. It turned out later that when following the approach to internal reliability (Prószyński 1994), these properties may help construct clearly interpretable reliability measures and reliability criteria for correlated observations. Following that approach, in the present study, the use is made of the observations and least squares residuals expressed as multiples of the a priori standard deviations. Moreover, one operates with the following three types of system responses to a single gross error: the local, the quasi-global and the global response, but within a different parameterization.

An attempt is also made to relate the proposed reliability measures to the reliability numbers, which together with statistical test parameters determine MDBs for individual observations. The problem of minimal detectable error or bias has been extensively investigated in geodetic literature (e.g. Baarda 1968; Van Mierlo 1978; Schaffrin 1997; Teunissen 1990, 1996, 1998), and the formulas derived so far, covering the case of uncorrelated and correlated observations, are considered in this paper as a reference.

\section{Basic notations and auxiliary formulas}

Let us consider a linear parametric model, written as

$\mathbf{A x}+\mathbf{e}=\mathbf{y} ; \quad \mathbf{e} \sim(\mathbf{0}, \mathbf{C})$

where $\mathbf{y}$ the $n \times 1$ vector of observations; $\mathbf{A}$ the $n \times u$ design matrix, rank $\mathbf{A}=u-d$ ( $d$, system defect, $d \geq 0) ; \mathbf{x}$ the unknown $u \times 1$ vector of parameters; e the unknown $n \times 1$ vector of random errors; $\mathbf{C}$ the $n \times n$ covariance matrix of $\mathbf{e}$ (positive definite).

The model (1) may also represent a linearized form of original non-linear model.

The disturbance/response relationship for the system (1) will be written as

$\Delta \mathbf{v}=-\mathbf{R} \cdot \Delta \mathbf{y}$

where $\Delta \mathbf{y}$ the vector of observation gross errors (disturbances), $\Delta \mathbf{v}$ the vector of increments in least squares residuals (responses), i.e. $\Delta \mathbf{v}=-\Delta \hat{\mathbf{e}}, \mathbf{R}$ the reliability matrix defined by
$\mathbf{R}=\mathbf{I}-\mathbf{A}\left(\mathbf{A}^{\mathrm{T}} \mathbf{C}^{-1} \mathbf{A}\right)^{-} \mathbf{A}^{\mathrm{T}} \mathbf{C}^{-1}$

where $(\cdot)^{-}$is $g$-inverse of $(\cdot)$.

The choice of $g$-inverse depends on the datum definition applied (Rao and Mitra 1971). Assuming the use of the minimum-constraints datum definition for $d>0$, the reflexive $g$-inverse is chosen. In the case where $d=0$, a regular inverse is used.

For a system with both correlated and uncorrelated observations $\mathbf{R}$ is an oblique projector. In regression analysis the projector $\mathbf{I}-\mathbf{R}$ is termed a hat matrix (Chatterjee and Hadi 1988).

To denote the elements of one-symbol matrices, the corresponding small letters will be used, e.g. $r_{i j}$ for $\{\mathbf{R}\}_{i j}$, whereas the elements of matrix expressions will be denoted with the use of parentheses, e.g. $\left\{\mathbf{R}^{\mathrm{T}} \mathbf{C}^{-1} \mathbf{R}\right\}_{i i}$.

\section{The modified reliability matrix and its main properties}

In order to establish a link with the approach to reliability measures for uncorrelated observations (Prószyński 1994), we shall modify the system (1) without affecting its least squares (LS) solution, by transforming the observations into the correlated dimensionless variables of equal accuracy.

Thus, multiplying both sides of the system (1) by $\sigma^{-1}$, where $\sigma=(\operatorname{diag} \mathbf{C})^{1 / 2}$, and transforming the covariance matrix $\mathbf{C}$ accordingly, we obtain a system

$\mathbf{A}_{s} \mathbf{x}+\mathbf{e}_{s}=\mathbf{y}_{s} ; \quad \mathbf{e}_{s} \sim\left(0, \mathbf{C}_{s}\right)$,

where $\mathbf{A}_{s}=\sigma^{-1} \mathbf{A}, \mathbf{e}_{s}=\sigma^{-1} \mathbf{e}, \mathbf{y}_{s}=\sigma^{-1} \mathbf{y}, \mathbf{C}_{\mathrm{s}}=\boldsymbol{\sigma}^{-1} \mathbf{C} \boldsymbol{\sigma}^{-1}$, $\mathbf{C}_{s}$ is a correlation matrix.

The vectors $\mathbf{e}_{s}$ and $\mathbf{y}_{s}$ are, respectively, the true errors and the observations expressed as multiples of the a priori standard deviations. For uncorrelated observations, we have $\mathbf{C}_{s}=\mathbf{I}$, where $\mathbf{I}$ is a unit matrix.

We omit the immediate proof that the modified system (4) is equivalent to the original system (1) with respect to the LS solution $\hat{\mathbf{x}}$. What is advantageous for reliability analysis of correlated observations is that by modifying the system (1) we are given a possibility of operating explicitly with the correlation matrix $\mathbf{C}_{S}$.

The disturbance/response relationship for the system (4) will take the form

$\Delta \mathbf{v}_{S}=-\mathbf{H} \cdot \Delta \mathbf{y}_{S}$,

where

$$
\begin{aligned}
\Delta \mathbf{y}_{s} & =\boldsymbol{\sigma}^{-1} \Delta \mathbf{y} ; \quad \Delta \mathbf{v}_{s}=\sigma^{-1} \Delta \mathbf{v} ; \quad \Delta \mathbf{v}_{s}=-\Delta \hat{\mathbf{e}}_{s} \\
\mathbf{H} & =\mathbf{I}-\mathbf{A}_{s}\left(\mathbf{A}_{s}^{\mathrm{T}} \mathbf{C}_{s}^{-1} \mathbf{A}_{s}\right)^{-} \mathbf{A}_{s}^{\mathrm{T}} \mathbf{C}_{s}^{-1}
\end{aligned}
$$


The relationship between $\mathbf{H}$ and $\mathbf{R}$ is as shown below

$$
\begin{aligned}
\mathbf{H} & =\mathbf{I}-\mathbf{A}_{s}\left(\mathbf{A}_{s}^{\mathrm{T}} \mathbf{C}_{s}^{-1} \mathbf{A}_{s}\right)^{-} \mathbf{A}_{s}^{\mathrm{T}} \mathbf{C}_{s}^{-1} \\
& =\mathbf{I}-\boldsymbol{\sigma}^{-1} \mathbf{A}\left(\mathbf{A}^{\mathrm{T}} \boldsymbol{\sigma}^{-1} \boldsymbol{\sigma} \mathbf{C}^{-1} \boldsymbol{\sigma} \boldsymbol{\sigma}^{-1} \mathbf{A}\right)^{-} \mathbf{A}^{\mathrm{T}} \boldsymbol{\sigma}^{-1} \boldsymbol{\sigma} \mathbf{C}^{-1} \boldsymbol{\sigma} \\
& =\boldsymbol{\sigma}^{-1}\left[\left(\mathbf{I}-\mathbf{A}\left(\mathbf{A}^{\mathrm{T}} \mathbf{C}^{-1} \mathbf{A}\right)^{-} \mathbf{A}^{\mathrm{T}} \mathbf{C}^{-1}\right)\right] \boldsymbol{\sigma}=\boldsymbol{\sigma}^{-1} \mathbf{R} \boldsymbol{\sigma}
\end{aligned}
$$

Here are the properties of the matrix $\mathbf{H}$ :

(a) $\mathbf{H}$ is similar to $\mathbf{R}$, and hence,

- since $\mathbf{R}$ is idempotent, $\mathbf{H}$ is also idempotent, i.e.

$$
\mathbf{H H}=\boldsymbol{\sigma}^{-1} \mathbf{R} \boldsymbol{\sigma} \cdot \boldsymbol{\sigma}^{-1} \mathbf{R} \boldsymbol{\sigma}=\boldsymbol{\sigma}^{-1} \mathbf{R} \mathbf{\sigma}=\boldsymbol{\sigma}^{-1} \mathbf{R} \boldsymbol{\sigma}=\mathbf{H}
$$

- $\operatorname{Tr} \mathbf{H}=\operatorname{Tr} \mathbf{R}$, i.e. $\operatorname{Tr} \mathbf{H}=\operatorname{Tr}\left(\boldsymbol{\sigma}^{-1} \mathbf{R} \boldsymbol{\sigma}\right)=\operatorname{Tr}(\mathbf{R} \boldsymbol{\sigma}$ $\left.\boldsymbol{\sigma}^{-1}\right)=\operatorname{Tr} \mathbf{R}$

- since $\sigma$ is diagonal, $h_{i i}=r_{i i}$ for $i=1, \ldots, n$, i.e. $h_{i i}=\sigma_{i}^{-1} r_{i i} \sigma_{i}=r_{i i}$.

(b) Since $\mathbf{H}$ is idempotent, but not symmetric (i.e. $\mathbf{H}^{\mathrm{T}} \neq$ H), it is an oblique projector (Rao and Mitra 1971).

(c) For uncorrelated observations, where $\mathbf{C}$ is diagonal and $\mathbf{C}_{S}=\mathbf{I}$, the matrix $\mathbf{H}$ takes the form, denoted here by $\overline{\mathbf{H}}$

$\overline{\mathbf{H}}=\mathbf{I}-\mathbf{A}_{s}\left(\mathbf{A}_{s}^{\mathrm{T}} \mathbf{A}_{s}\right)^{-} \mathbf{A}_{s}^{\mathrm{T}}$

or equivalently $\overline{\mathbf{H}}=\mathbf{I}-\boldsymbol{\sigma}^{-1} \mathbf{A}\left(\mathbf{A}^{\mathrm{T}} \boldsymbol{\sigma}^{-2} \mathbf{A}\right)^{-} \mathbf{A}^{\mathrm{T}} \boldsymbol{\sigma}^{-1}$, where $\boldsymbol{\sigma}=\mathbf{C}^{1 / 2}, \overline{\mathbf{H}}$ is idempotent and symmetric, and hence it is an orthogonal projector (Rao and Mitra 1971)

- $\operatorname{since} \operatorname{rank} \overline{\mathbf{H}}=\operatorname{rank} \mathbf{H}=n-u+d$ and $\overline{\mathbf{H}}$ is idempotent, we have $\operatorname{Tr} \overline{\mathbf{H}}=\operatorname{Tr} \mathbf{H}$.

(d) Let the asymmetry of $\mathbf{H}$ be expressed by

$\mathbf{U}=\mathbf{H}-\mathbf{H}^{\mathrm{T}}$

or, on the basis of (7), equivalently

$\mathbf{W}=\mathbf{U H}=\mathbf{H}-\mathbf{H}^{\mathrm{T}} \mathbf{H}$.

We may notice that, since $\mathbf{H}^{\mathrm{T}} \mathbf{W}=\mathbf{H}^{\mathrm{T}} \mathbf{H}-\mathbf{H}^{\mathrm{T}} \mathbf{H}=\mathbf{0}$, each column of $\mathbf{W}$ is a vector belonging to the null-space of $\mathbf{H}^{\mathrm{T}}$.

(e) From the idempotency of $\mathbf{H}$ it follows, that for every diagonal element $h_{i i}(i=1, \ldots, n)$ the following relationship holds true

$$
\sum_{k=1}^{k=n}\left(h_{i k} \cdot h_{k i}\right)=h_{i i}
$$

and finally, after a simple rearrangement of terms

$h_{i i}^{2}-h_{i i}+\sum_{k \neq i}\left(h_{i k} \cdot h_{k i}\right)=0$.

For the roots of this quadratic trinomial to be real numbers, it is necessary that

$\sum_{k \neq i}\left(h_{i k} \cdot h_{k i}\right) \leq 0.25$.

On the basis of (10), we may note that with $h_{i i}=0$ there holds an equality

$\sum_{k \neq i}\left(h_{i k} \cdot h_{k i}\right)=0$

(f) auxiliary relationships (proofs consist of a series of operations with the use of the formula for $\mathbf{H}$ as in (5))

$$
\mathbf{H}^{\mathrm{T}} \mathbf{C}_{s}^{-1} \mathbf{H}=\mathbf{C}_{s}^{-1} \mathbf{H} \quad \mathbf{H C}_{s} \mathbf{H}^{\mathrm{T}}=\mathbf{H C}_{s}
$$

\section{Responses of the system to a single disturbance}

Considering the case of correlated observations, we shall use the same types of system responses to a single standardized gross error as were introduced in Prószyński (1994) for systems with uncorrelated observations, i.e. the responses: $\mathrm{L}$ (local), Q (quasi-global) and G (global).

We shall assume a unit gross error in the $i$-th observation, i.e.

$\Delta \mathbf{y}_{s}^{\mathrm{T}}=\left[\begin{array}{lllllll}0 & \ldots & 0 & \Delta_{s, i} & 0 & \ldots & 0\end{array}\right]^{\mathrm{T}}, \quad$ where $\Delta_{s, i}=1$.

On the basis of the disturbance/response relationship (5), we obtain

$$
\begin{aligned}
L_{i(i)} & =-h_{i i} \quad Q_{(i)}=\sqrt{\sum_{k=1, k \neq i}^{n} h_{k i}^{2}} \\
G_{(i)} & =\sqrt{\sum_{k=1}^{n} h_{k i}^{2} .}
\end{aligned}
$$

The responses are interrelated by the equality

$L_{i(i)}^{2}+Q_{(i)}^{2}=G_{(i)}^{2}$.

We shall now link each of the responses $Q_{(i)}$ and $G_{(i)}$ with the response $L_{i(i)}$.

On the basis of (10) we get

$\sum_{k \neq i}\left(h_{i k} \cdot h_{k i}\right)=h_{i i}-h_{i i}^{2}$. 
Introducing the non-diagonal element $u_{i k}$ of the matrix $\mathbf{U}$ (see (7)) as an auxiliary quantity

$u_{i k}=h_{i k}-h_{k i}(k \neq i)$,

we can write

$h_{i k}=h_{k i}+u_{i k}$

and after substituting into (16) and carrying out some simple operations we obtain

$\sum_{k \neq i} h_{k i}^{2}+\sum_{k \neq i} h_{k i} u_{i k}=h_{i i}-h_{i i}^{2}$.

Since $\sum_{k \neq i} h_{k i}^{2}=Q_{(i)}^{2}$ and $\sum_{k \neq i} h_{k i} u_{i k}=\sum_{k} h_{k i} u_{i k}=$ $w_{i i}$ (see formula (8)) we get

$Q_{(i)}^{2}+w_{i i}=h_{i i}-h_{i i}^{2}$

and after rearranging the terms, we obtain finally

$Q_{(i)}=\sqrt{h_{i i}-h_{i i}^{2}-w_{i i}}$

where, according to (8), $w_{i i}$ may also be written as

$w_{i i}=h_{i i}-\left\{\mathbf{H}^{\mathrm{T}} \mathbf{H}\right\}_{i i}$

The term $w_{i i}$ can be interpreted as an effect of asymmetry of the $i$-th row and the $i$-th column of the matrix $\mathbf{H}$. With $w_{i i}=0$ the formula (19) takes the form identical to that for systems with uncorrelated observations.

From the definition (14) and the Eq. (19) we get the response $G_{(i)}$ expressed also in terms of $h_{i i}$ and $w_{i i}$

$G_{(i)}=\sqrt{h_{i i}-w_{i i}}$.

Taking into account (20) the responses $Q_{(i)}$ and $G_{(i)}$ as in (19) and (21) may be written in an equivalent form.

$Q_{(i)}=\sqrt{\left(\mathbf{H}^{\mathrm{T}} \mathbf{H}\right)_{i i}-h_{i i}^{2}} \quad G_{(i)}=\sqrt{\left(\mathbf{H}^{\mathrm{T}} \mathbf{H}\right)_{i i}}$

\section{Proposed measures of internal reliability}

From the previous section it follows that the responses to a single gross error in the $i$-th observation, having the form

$L_{i(i)}=-h_{i i} \quad Q_{(i)}=\sqrt{h_{i i}-h_{i i}^{2}-w_{i i}} \quad G_{(i)}=\sqrt{h_{i i}-w_{i i}}$

are defined by two parameters; $h_{i i}$ and $w_{i i}$.

This necessitates the use of a two-parameter reliability measure for the $i$-th observation

$h_{(i)}=\left(h_{i i}, w_{i i}\right)$.

For the systems with uncorrelated observations, we have $w_{i i}=0$ and hence $h_{(i)}=\left(\bar{h}_{i i}, 0\right)$, or simply $h_{(i)}=\bar{h}_{i i}$, where $\bar{h}_{i i}$ is the diagonal element of the matrix $\overline{\mathbf{H}}$ (see (6)).

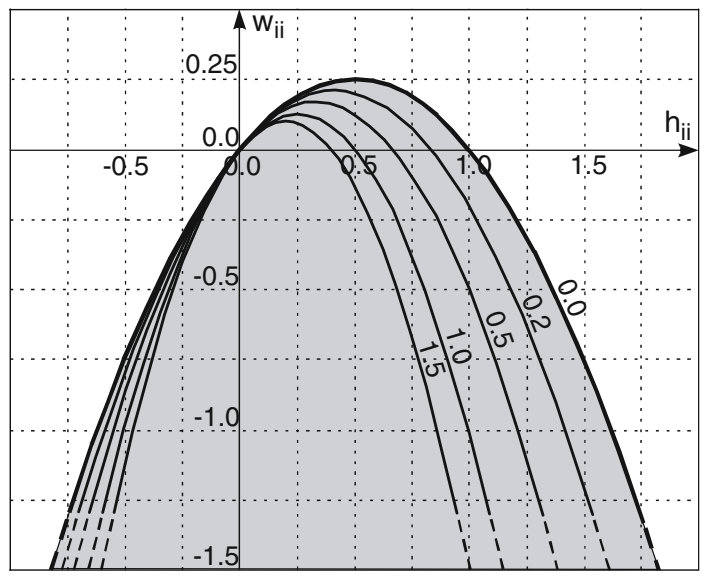

Fig. 1 The range of values for the reliability parameters $h_{i i}$ and $w_{i i}$

The two-parameter measure (24) can therefore be considered a generalized (with respect to correlation of observations) reliability measure for an individual observation.

The parameters $h_{i i}$ and $w_{i i}$ are mutually related. We shall determine this relationship based on definitions of the responses $Q_{(i)}$ and $G_{(i)}$ given by the formulas (14).

For $Q_{(i)}$ as in (23) we shall write

$h_{i i}-h_{i i}^{2}-w_{i i} \geq 0$

and hence

$w_{i i} \leq h_{i i}-h_{i i}^{2}$.

It is easy to check that the condition for the response $G_{(i)}$, being $w_{i i} \leq h_{i i}$, is satisfied with the inequality (26) as well.

The resulting range of values for $h_{i i}$ and $w_{i i}$ is indicated in Fig. 1 by a grey area.

A characteristic quantity in reliability analysis is also a ratio of the responses $Q_{(i)}$ and $\left|L_{i(i)}\right|$. Let us then introduce a coefficient $k_{i}$, defined as

$$
\begin{aligned}
k_{i} & =\frac{Q_{(i)}^{2}}{L_{i(i)}^{2}}=\frac{h_{i i}-h_{i i}^{2}-w_{i i}}{h_{i i}^{2}} \\
& =\frac{1}{h_{i i}}-\frac{w_{i i}}{h_{i i}^{2}}-1 \quad\left(\text { for }_{i i} \neq 0\right) .
\end{aligned}
$$

Transforming (27) we may arrive at the equations of the lines $k_{i}=$ const., i.e.

$w_{i i}=h_{i i}-\left(1+k_{i}\right) h_{i i}^{2}$.

The lines for $k_{i}=0.0,0.2,0.5,1.0,1.5$ are shown in Fig. 1 .

To provide a complementary representation to that in Fig. 1, we see in Fig. 2 the diagrams for the relationship (27).

To present in graphical form the responses $L_{i(i)}, Q_{(i)}$ and $G_{(i)}$ being the functions of $h_{i i}$ and $w_{i i}$ (see (23)), in Fig. 3 are shown the cross sections of these surfaces for some selected values of $w_{i i}$, i.e. $0.25,0.2,0.1,0,-0.3,-0.6,-1.0$. 


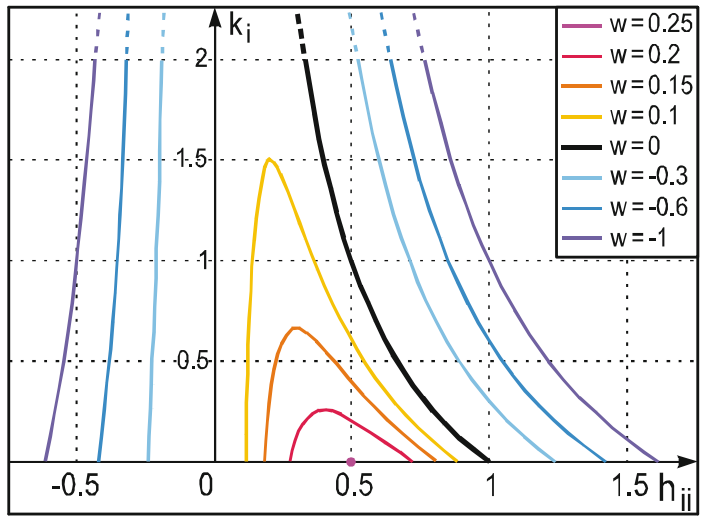

Fig. 2 The lines $w_{i i}=$ const. for the relationship $k_{i}=f\left(h_{i i}, w_{i i}\right)$

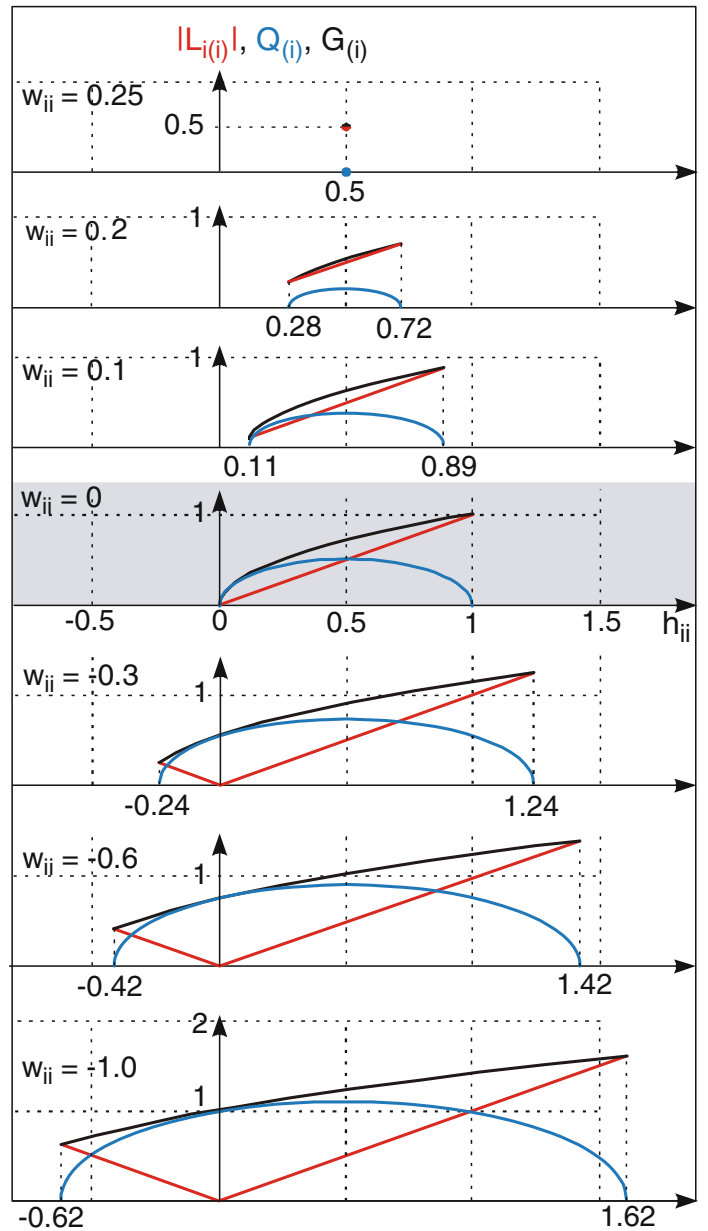

Fig. 3 The responses of the system for selected values of the parameter $w_{i i}$

For $w_{i i}=0$, which is the case for uncorrelated observations, we get curves that correspond to those shown in Prószyński (1994). Identical curves would be obtained, if instead of $h_{i i}$ we used $z_{i}=\sqrt{h_{i i}}$.

To assess the effects of correlation in a particular observation system it is useful to confront the reliability characteris- tics $\left(h_{i i}, w_{i i}\right)$ and $\left(L_{i(i)}, k_{i}\right)$ with the quantities $\bar{h}_{i i}$, obtained after omitting the correlation.

The correlation between the observations replaces the values of $\bar{h}_{i i}$ with the values of $h_{i i}$ and $w_{i i}$, for $i=1, \ldots, n$. Since $\operatorname{Tr} \overline{\mathbf{H}}=\operatorname{Tr} \mathbf{H}$ it follows that $\sum_{n} h_{i i}=\sum_{n} \bar{h}_{i i}$, which means that correlation changes the local responses so that their sum remains constant. In view of that, the system should be designed with a certain reliability surplus in the values of $\bar{h}_{i i}$.

The correlation also causes asymmetry in the operator $\mathbf{H}$, reflected in the parameter $w_{i i}$. On the basis of the following partial derivatives, obtained from (23) and (27)

$$
\begin{aligned}
\frac{\partial G_{(i)}}{\partial w_{i}} & =-\frac{1}{2 G_{(i)}} ; \quad \frac{\partial Q_{(i)}}{\partial w_{i}}=-\frac{1}{2 Q_{(i)}} ; \\
\frac{\partial k_{i}}{\partial w_{i}} & =-\frac{1}{h_{i i}^{2}} \quad \text { for } h_{i i} \neq 0
\end{aligned}
$$

the role of asymmetry can be characterized as below: with increasing values of $w_{i i}$ (see Fig. 3):

- both the global response and the quasi-global response become smaller;

- the relation of the quasi-global response to the local response becomes smaller and this effect is especially intense for small values of $\left|h_{i i}\right|$ (see Fig. 1).

With respect to the effectiveness of outlier detection we can consider the first effect as disadvantageous while the second one as advantageous. Hence, the case of $w_{i i}=0$ would indicate a compromise.

\section{Criteria for internal reliability}

A reasonable postulate concerning the behaviour of a system in the presence of single gross error can be that maximum response should be located in the observation in which the error resides, and that the responses in other observations should possibly be the smallest. If a system is structured so that it can respond in that manner, the chances of effective outlier detection are expected to be greater.

In analogy to criteria set for uncorrelated observations, we shall then require that:

- the local response $L_{i(i)}$ should compensate for at least half of the disturbance $\Delta_{s, i}$, i.e.

$0<\Delta_{s, i}+L_{i(i)}<0.5 \cdot \Delta_{s, i}$,

where $L_{i(i)}=-h_{i i} \Delta_{s, i}$ 


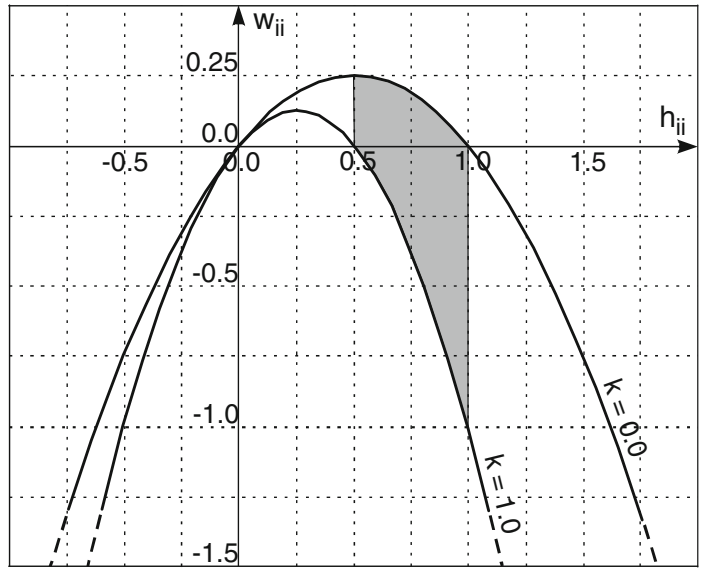

Fig. 4 The area where the proposed reliability criteria are met

- the local response $L_{i(i)}$ with its absolute value should surpass the quasi-global response $Q_{(i)}$, i.e.

$0<k_{i}<1$,

where $k_{i}$ as in (27).

The above criteria, when expressed in terms of the reliability parameters $h_{i i}$ and $w_{i i}$, take the following form

$0.5<h_{i i} \leq 1 \cap h_{i i}-2 h_{i i}^{2}<w_{i i}<h_{i i}-h_{i i}^{2} \quad i=1, \ldots, n$

The area corresponding to the proposed reliability criteria is shown in Fig. 4.

In systems with uncorrelated observations, the fulfilment of the first requirement (29) implies automatically the fulfilment of the second requirement (30).

For systems which do not fulfil the reliability criteria $\bar{h}_{i i}>$ $0.5 i=1, \ldots, n$ (i.e. when neglecting the correlation) the criteria as in (31) cannot be met for all observations. This follows immediately from the property $\sum h_{i i}=\sum \bar{h}_{i i}$. If we have such weak systems, and high correlation between the observations is unavoidable, less rigorous criteria than those in (31) may be used. However, we must be aware of the difficulties in detecting potential outliers.

The weaker criteria might, for instance, read as follows:

$\left|\Delta_{s, i}+L_{i(i)}\right|<0.5 \cdot \Delta_{s, i}$ and $0<k_{i}<1.2$

or in terms of $h_{i i}$ and $w_{i i}$

$0.5<h_{i i} \leq 1.5 \cap h_{i i}-2.2 h_{i i}^{2}<w_{i i}<h_{i i}-h_{i i}^{2}$

The proposed two-dimensional reliability criteria classify the measures $h_{(i)}=\left(h_{i i}, w_{i i}\right) i=1, \ldots, n$, into those which are acceptable and those which are unacceptable. Ranking of the measures, which would impose one-dimensional hierarchy, might enable one to make comparisons between the measures for individual observations. This turned out to be too complicated even for the group of acceptable measures.
The interrelation between the parameters $h_{i i}$ and $w_{i i}$ was one of the causes. So we limit ourselves in this situation to the statement that within the measures that meet the reliability criteria (31) preference should be given to those which are closer to the measures preferred in the case of uncorrelated observations. We mean here such $h_{(i)}$, in which $h_{i i}$ is of high value and $\left|w_{i i}\right|$ is of small value. This implies that among the measures for the $i$-th and the $j$-th observation which meet the criteria (31), such that

$h_{i i}>h_{j j} \cap\left|w_{i i}\right|<\left|w_{j j}\right|$

the measure for the $i$-th observation will be preferred. And consequently, the $i$-th observation will be considered of higher reliability than the $j$-th one.

\section{Reliability numbers as expressed in terms of minimal detectable bias}

Let us recall the formula for the minimal detectable bias for the $i$-th observation (Teunissen 1990, 1996), containing the general expression for reliability number $r_{i}$, covering the case of correlated and uncorrelated observations. We shall adjust its form to modified system (4) and the notation used in the present paper.

Since the following relation can be proved

$\frac{1}{\sigma_{i}^{2}}\left\{\mathbf{H}^{\mathrm{T}} \mathbf{C}_{s}^{-1} \mathbf{H}\right\}_{i i}=\left\{\mathbf{R}^{\mathrm{T}} \mathbf{C}^{-1} \mathbf{R}\right\}_{i i}$,

where $\mathbf{C}, \mathbf{R}$ are the quantities for the original system (1), we obtain

$\operatorname{MDB}_{i}=\sigma_{i} \sqrt{\frac{\lambda}{r_{i}}} \quad r_{i}=\left\{\mathbf{H}^{\mathrm{T}} \mathbf{C}_{s}^{-1} \mathbf{H}\right\}_{i i}=\cos ^{2} \alpha_{\mathrm{s}, \mathrm{i}}\left\{\mathbf{C}_{s}^{-1}\right\}_{i i}$,

where $\lambda$ the noncentrality parameter, $\sigma_{i}$ the standard deviation of the $i$-th observation, $\alpha_{s, i}$ the angle between the unit vector for the $i$-th standardized observation and the orthogonal complement of the range space of the matrix $\mathbf{A}_{s}$. We can check that $\cos ^{2} \alpha_{s, i}=\cos ^{2} \alpha_{i}$, where $\alpha_{i}$ is the equivalently defined angle for the original system.

For uncorrelated observations we obtain $r_{i}=\cos ^{2} \alpha_{s, i}$ or equivalently $r_{i}=\left\{\mathbf{H}^{\mathrm{T}} \mathbf{C}_{s}^{-1} \mathbf{H}\right\}_{i i}=\{\overline{\mathbf{H}} \mathbf{I} \overline{\mathbf{H}}\}_{i i}=\{\overline{\mathbf{H}}\}_{\mathrm{ii}}=\overline{\mathrm{h}}_{\mathrm{ii}}$, where $r_{i}\langle 0,1)$ is a redundancy number. For correlated observations $r_{i}$ as in (34), having the range $\langle 0, \infty)$, corresponds to "generalized reliability number" (Wang and Chen 1994). Without using another interpretable angle $\beta_{\mathrm{i}}$ as in Teunissen $(1990,1996)$ the "normalized reliability number" $r_{i}^{\prime}\langle 0,1)$, proposed by Schaffrin (1997), can be written in the form

$r_{i}^{\prime}=\frac{r_{i}}{\left\{\mathbf{C}_{s}^{-1}\right\}_{i i}}=\cos ^{2} \alpha_{s, i}$ 
The formula shows that the normalization factor as based only on the elements of correlation matrix $\mathbf{C}_{S}$ without taking into account the design matrix $\mathbf{A}_{s}$, although bringing clear and useful geometric interpretation of $r_{i}^{\prime}$, may not guarantee the monotony of mapping $r_{i} \rightarrow r_{i}^{\prime}$ (see Sect.9). Leaving this problem for separate analysis, we notice that neither $r_{i}$ nor $r_{i}^{\prime}$ may indicate the observations where the system displays an abnormal response to the unit gross error, i.e. $h_{i i}<0$ or $h_{i i}>1$.

That is why the proposed two-dimensional reliability measure $h_{(i)}$ as in (24), containing the redundancy number $h_{i i}$ seems to be more informative for design purposes. However, with $h_{(i)}$ we practically lose the possibility of ranking the observations in terms of reliability.

We shall now discuss the relation between $G_{(i)}^{2}=\left\{\mathbf{H}^{\mathrm{T}} \mathbf{H}\right\}_{i i}$ and $r_{i}=\left\{\mathbf{H}^{\mathrm{T}} \mathbf{C}_{s}^{-1} \mathbf{H}\right\}_{i i}$, being the quantities of global character. With the unit gross error, i.e. $\Delta_{s, i}=1$ as in (13):

- $\left\{\mathbf{H}^{\mathrm{T}} \mathbf{C}_{s}^{-1} \mathbf{H}\right\}_{i i}$ is a sum of squares of responses in the quantities being linear functions of original observations, the functions depending on the decorrelation procedure used. Hence, we may have different sets of responses with the same sum of their squares. The sum cannot be decomposed so that a return to responses in original observations is achieved;

- $\left\{\mathbf{H}^{\mathrm{T}} \mathbf{H}\right\}_{i i}$ is a sum of squares of responses in original observations (only scaled in the a priori standard deviations), and hence the responses are unique. Compared to the former sum, this is not meaningful with respect to outlier detection, since it neglects the correlation between the responses in individual observations.

To explain it more thoroughly, we shall introduce the vector $\mathbf{H}_{\text {. }}$ being the i-th column of the matrix $\mathbf{H}$. We notice that $\left\{\mathbf{H}^{\mathrm{T}} \mathbf{C}_{s}^{-1} \mathbf{H}\right\}_{\mathrm{ii}}$ is the square of the norm of the vector $\mathbf{H}_{\mathrm{i}}$ in a space with the metric of $\mathbf{C}_{s}$, whereas $\left\{\mathbf{H}^{\mathrm{T}} \mathbf{H}\right\}_{i i}$ is the square of the norm of the same vector $\mathbf{H}_{. i}$ in the Euclidean space (using the analogy-with the metric of $\mathbf{C}_{s}=\mathbf{I}$ ). Since no relation between $\mathbf{C}_{S}$ and $\mathbf{I}$ can be formulated (unlike for $\mathbf{C}$ and $\mathbf{C}_{S}$ ), no relationship between the two expressions can be established.

So, the aforementioned two types of global responses differ in meaning, and consequently, in value. It is only for the case of uncorrelated observations that they are of equal values, as each of them becomes a redundancy number $\bar{h}_{i i}$. Numerical tests carried out so far have not disclosed any regularities of general character between these two quantities.

Thus, the global response $G_{(i)}^{2}=\left\{\mathbf{H}^{\mathrm{T}} \mathbf{H}\right\}_{i i}$, as different from the redundancy number $r_{i}=\left\{\mathbf{H}^{\mathrm{T}} \mathbf{C}_{s}^{-1} \mathbf{H}\right\}_{i i}$, is not related to $\mathrm{MDB}$, which is a weakness of the proposed approach.

\section{The case of specific correlation structure}

Let us consider the system (4) with a specific correlation matrix $\mathbf{C}_{s}$ and the reliability matrix $\mathbf{H}$ expressed in a corresponding block structure, as shown below

$\mathbf{C}_{s}=\left[\begin{array}{ll}\mathbf{I}_{1} & \mathbf{0} \\ \mathbf{0}^{\mathrm{T}} & \mathbf{C}_{s, 2}\end{array}\right] \quad \mathbf{H}=\left[\begin{array}{ll}\mathbf{H}_{11} & \mathbf{H}_{12} \\ \mathbf{H}_{21} & \mathbf{H}_{22}\end{array}\right]$

where $\mathbf{I}_{1}$ and $\mathbf{H}_{11}$ are the $(p \times p)$ matrices.

To obtain the reliability numbers $r_{i}$ as in (35), we find the matrix $\mathbf{C}_{s}^{-1} \mathbf{H}=\mathbf{H}^{\mathrm{T}} \mathbf{C}_{s}^{-1} \mathbf{H}$ (see (12))

$$
\begin{aligned}
\mathbf{C}_{s}^{-1} \mathbf{H} & =\left[\begin{array}{ll}
\mathbf{I}_{1} & \mathbf{0} \\
\mathbf{0}^{\mathrm{T}} & \mathbf{C}_{s, 2}^{-1}
\end{array}\right]\left[\begin{array}{ll}
\mathbf{H}_{11} & \mathbf{H}_{12} \\
\mathbf{H}_{21} & \mathbf{H}_{22}
\end{array}\right] \\
& =\left[\begin{array}{ll}
\mathbf{H}_{11} & \mathbf{H}_{12} \\
\mathbf{C}_{s, 2}^{-1} \mathbf{H}_{21} & \mathbf{C}_{s, 2}^{-1} \mathbf{H}_{22}
\end{array}\right]
\end{aligned}
$$

We can see that for a subset of $p$ uncorrelated observations we have $\left(\mathbf{C}_{s}^{-1} \mathbf{H}\right)_{11}=\mathbf{H}_{11}$ and thus $\left\{\mathbf{C}_{s}^{-1} \mathbf{H}\right\}_{i i}=\{\mathbf{H}\}_{i i}=$ $h_{i i}, i=1, \ldots, p$, which can be extended upon a more complex correlation structure (i.e. with a greater number of subsets of uncorrelated and correlated observations).

This can be proof of the property that for every subset of uncorrelated observations co-existing in a system with correlated observations, the reliability numbers are equal to redundancy numbers.

Assuming the same block structure of $\mathbf{C}_{S}$ and $\mathbf{H}$ as in (36) we can also prove that $\left(\mathbf{H C}_{S}\right)_{11}=\mathbf{H}_{11}$, which yields

$\left\{\mathbf{H C}_{s}\right\}_{i i}=\{\mathbf{H}\}_{i i}=h_{i i}, \quad i=1, \ldots, p$,

where $\left\{\mathbf{H C}_{s}\right\}_{i i}=\sigma_{\mathbf{v}_{s, i}}^{2}$, i.e. the variance of the $i$-th residual in the system (4).

\section{Numerical examples}

Example 1 The task is to carry out the analysis of internal reliability for a simulated test network shown in Fig. 5. It is a linear-angular network $(L, \alpha)$ strengthened by GPS vectors $(\Delta X, \Delta Y)$. The $(L, \alpha)$ observations are uncorrelated, and $\sigma_{L}=(1+1 \mathrm{ppm})[\mathrm{mm}], \sigma_{\alpha}=3^{c c}$. The covariance matrix for GPS vectors $12-11,12-1,12-5,12-13$ is shown after Tables 1 and 2.

Table 1 Approximate coordinates

\begin{tabular}{lcr}
\hline Point no. & $\mathrm{X}^{o}(\mathrm{~m})$ & $\mathrm{Y}^{o}(\mathrm{~m})$ \\
\hline 1 & 1,317 & 1,133 \\
5 & 1,193 & 1,387 \\
11 & 1,098 & 801 \\
12 & 890 & 1,095 \\
13 & 796 & 1,598 \\
\hline
\end{tabular}


Table 2 The results of internal reliability analysis for the test network

\begin{tabular}{lllllllll}
\hline $\begin{array}{l}\text { Observation L, } \\
\alpha(L-\mathrm{R}-\mathrm{C}) \Delta X, \Delta Y\end{array}$ & $\bar{h}_{i i}$ & \multicolumn{1}{l}{$h_{i i}$} & \multicolumn{1}{l}{$w_{i i}$} & $k_{i}$ & Criteria + - & $G_{(i)}^{2}$ & $r_{i}$ & $r_{i}^{\prime}$ \\
\hline$L 11-1$ & & & & & & & & \\
\hline 11-5 & 0.442 & 0.450 & 0.004 & 1.20 & - & 0.447 & 0.450 & 0.450 \\
$L 1-5$ & 0.489 & 0.462 & -0.038 & 1.35 & - & 0.500 & 0.462 & 0.462 \\
$L 5-13$ & 0.523 & 0.548 & 0.022 & 0.75 & + & 0.526 & 0.548 & 0.548 \\
$L 11-13$ & 0.496 & 0.521 & 0.021 & 0.84 & + & 0.500 & 0.521 & 0.521 \\
$L 1-13$ & 0.620 & 0.634 & 0.002 & 0.57 & + & 0.632 & 0.634 & 0.634 \\
$L 11-12$ & 0.581 & 0.597 & 0.009 & 0.65 & + & 0.588 & 0.597 & 0.597 \\
$L 12-13$ & 0.506 & 0.546 & 0.026 & 0.74 & + & 0.519 & 0.546 & 0.546 \\
$\alpha 5-13-12$ & 0.561 & 0.550 & -0.017 & 0.87 & + & 0.567 & 0.550 & 0.550 \\
$\alpha 11-1-12$ & 0.711 & 0.692 & -0.027 & 0.50 & + & 0.719 & 0.692 & 0.692 \\
$\alpha 1-5-12$ & 0.626 & 0.608 & -0.023 & 0.71 & + & 0.631 & 0.608 & 0.608 \\
\hline$\Delta X 12-11$ & 0.749 & 0.734 & -0.028 & 0.41 & + & 0.762 & 0.734 & 0.734 \\
$\Delta Y 12-11$ & 0.635 & 0.598 & -0.050 & 0.81 & + & 0.648 & 0.632 & 0.551 \\
$\Delta X 12-1$ & 0.734 & 0.758 & -0.063 & 0.43 & + & 0.821 & 0.890 & 0.773 \\
$\Delta Y 12-1$ & 0.466 & 0.455 & -0.023 & 1.31 & - & 0.478 & 0.512 & 0.447 \\
$\Delta X 12-5$ & 0.571 & 0.568 & -0.025 & 0.84 & + & 0.593 & 0.673 & 0.568 \\
$\Delta Y 12-5$ & 0.549 & 0.527 & -0.035 & 1.02 & - & 0.562 & 0.570 & 0.489 \\
$\Delta X 12-13$ & 0.548 & 0.519 & -0.063 & 1.16 & - & 0.582 & 0.583 & 0.482 \\
$\Delta Y 12-13$ & 0.428 & 0.451 & 0.002 & 1.21 & - & 0.449 & 0.562 & 0.457 \\
\hline & 0.765 & 0.782 & -0.012 & 0.30 & + & 0.794 & 0.887 & 0.792 \\
\hline & & & & & & & &
\end{tabular}

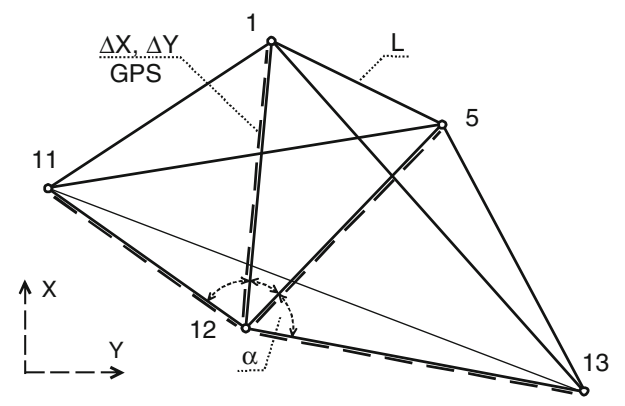

Fig. 5 The test network

$$
\begin{aligned}
& \mathbf{C}_{\text {GPS }} \\
& =\left[\begin{array}{rrrrrrrr}
4.50 & 1.00 & 0.40 & -0.35 & 0.25 & -0.20 & 0.20 & -0.15 \\
1.00 & 3.20 & 0.80 & 0.50 & -0.30 & 0.25 & -0.25 & 0.20 \\
0.40 & 0.80 & 3.00 & 0.70 & -0.25 & 0.20 & -0.15 & 0.15 \\
-0.35 & 0.50 & 0.70 & 2.90 & 0.65 & 0.35 & -0.30 & 0.25 \\
0.25 & -0.30 & -0.25 & 0.65 & 4.30 & 0.90 & 0.20 & -0.20 \\
-0.20 & 0.25 & 0.20 & 0.35 & 0.90 & 2.70 & 0.80 & 0.30 \\
0.20 & -0.25 & -0.15 & -0.30 & 0.20 & 0.80 & 3.30 & 1.20 \\
-0.15 & 0.20 & 0.15 & 0.25 & -0.20 & 0.30 & 1.20 & 5.00
\end{array}\right]\left[\mathrm{mm}^{2}\right]
\end{aligned}
$$

The results of the analysis carried out with the assumption that point 12 is fixed are presented in Table 2 and also in graphical form in two representations (Fig. 6). In table, in addition to the proposed reliability measures $\left(h_{i i}, w_{i i}\right.$ and $\left.k_{\mathrm{i}}\right)$ and auxiliary characteristics $\left(\bar{h}_{i i}, G_{(i)}^{2}=\left\{\mathbf{H}^{\mathrm{T}} \mathbf{H}\right\}_{i i}\right)$, the values
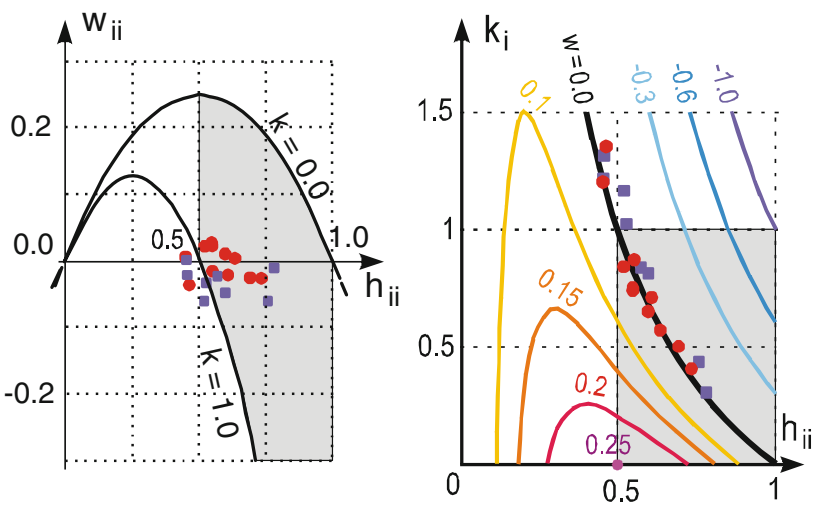

- $\mathrm{L}, \alpha$

- $\Delta \mathrm{X}, \Delta \mathrm{Y}$

Fig. 6 Graphs showing results of the reliability analysis for a test network

of the existing reliability measures, i.e. $r_{i}=\left\{\mathbf{H}^{\mathrm{T}} \mathbf{C}_{s}^{-1} \mathbf{H}\right\}_{i i}$ and $r_{i}^{\prime}=\left\{\mathbf{H}^{\mathrm{T}} \mathbf{C}_{s}^{-1} \mathbf{H}\right\}_{i i} /\left\{\mathbf{C}_{s}^{-1}\right\}_{i i}$, are shown to enable comparisons. To facilitate reading of the table, the correlated observations are separated from the uncorrelated observations by a bold line.

With $w_{i i}$ being of small absolute values, the plots in Fig. 6 are concentrated along the line $w_{i i}=0$.

Six of them are outside the acceptable area defined by the proposed reliability criteria. Hence, the network needs 
Table 3 The results of internal reliability analysis for a leveling network

\begin{tabular}{llrlllllll}
\hline Observation & $\bar{h}_{i i}$ & \multicolumn{1}{c}{$h_{i i}$} & $w_{i i}$ & $k_{i}$ & Criteria + /- & $G_{(i)}^{2}$ & $r_{i}$ & $r_{i}^{\prime}$ & MDB $_{i}^{*}$ \\
\hline $1-2$ & 0.250 & -1.00 & -3.40 & 1.40 & - & 2.40 & 2.00 & 0.11 \\
$2-3$ & 0.125 & 0.50 & -0.70 & 3.80 & - & 1.20 & 1.00 & 0.50 \\
$3-1$ & 0.625 & 1.50 & -4.50 & 1.67 & - & 6.00 & 5.00 & 0.25 & 1.00 \\
\hline
\end{tabular}

improvement. The maximum discrepancy between the values of $G_{(i)}^{2}$ and $r_{i}$, as referred to the letter values, is $-20.1 \%$ (for the observation $\Delta X 12-13$ ). We can also see that for two pairs of observations, i.e. $\Delta X 12-5, \Delta Y 12-5$ and $\Delta Y 12-11$, $\Delta Y 12-13$, the mapping $r_{i} \rightarrow r_{i}^{\prime}$ yields a reverse ranking.

Example 2 To demonstrate how mutually dispersed values the proposed reliability characteristics may display in the case of badly conditioned covariance matrices of observations, we show the results of the analysis for a simple levelling network used as a study case in (Wang and Chen 1994) and (Schaffrin 1997):

$$
\begin{aligned}
\mathbf{A} & =\left[\begin{array}{rr}
1 & 0 \\
-1 & 1 \\
0 & 1
\end{array}\right] \quad \mathbf{C}=\left[\begin{array}{lll}
2 & 0 & 3 \\
0 & 1 & 0.5 \\
3 & 0.5 & 5
\end{array}\right], \\
\mathbf{C}_{s} & =\left[\begin{array}{lll}
1 & 0 & 0.95 \\
0 & 1 & 0.22 \\
0.95 & 0.22 & 1
\end{array}\right]
\end{aligned}
$$

The eigenvalues of $\mathbf{C}: \lambda_{1}=0.069 ; \lambda_{2}=1.046 ; \lambda_{3}=6.885$.

Beside all the quantities listed in Table 2 (Example 1), Table 3 shows the $\mathrm{MDB}_{i}^{*}$ values for extended analysis, where $\mathrm{MDB}_{i}^{*}=\mathrm{MDB}_{i} / \sqrt{\lambda}$.

Neither of the observations satisfy the proposed reliability criteria. Due to correlation, the network responses in the observations 1-2 and 3-1 are abnormal, since for 1-2 the response instead of compensating for the disturbance enlarges it and for 3-1 the disturbance is compensated for but excessively. Similar to Example 1, the mapping $r_{i} \rightarrow r_{i}^{\prime}$ changes the ranking of observations to an even greater extent. However, the ranking of $r_{i}^{\prime}$ values seems to be more appropriate as it gives priority to the observation 2-3 displaying the normal behaviour of the analysed network. The MDB values do not show any differentiation.

The discrepancies between the values of $\left\{\mathbf{H}^{\mathrm{T}} \mathbf{H}\right\}_{i i}$ and $\left\{\mathbf{H}^{\mathrm{T}} \mathbf{C}_{s}^{-1} \mathbf{H}\right\}_{i i}$ amount to $+20 \%$ for each of the three observations.

\section{Conclusions}

The findings of this paper confirm the conclusions drawn from the solutions presented in Wang and Chen (1994), Schaffrin (1997) and Prószyński (1998), that the internal reliability for systems with correlated observations cannot be fully described by means of one-dimensional quantities with $\langle 0,1\rangle$ domain each. Therefore, the two-parameter reliability measures have been proposed, without setting any arbitrary restrictions upon the ranges of their values. They provide insight into the structure of network responses to gross errors, the responses referring to original observations, when scaled only with their a priori standard deviations. The advantage of the approach is that it offers reliability criteria interpretable in terms of network responses to disturbances. This is of great importance for the phase of network design. Weaknesses of the proposed measures are that they cannot provide a basis for determination of MDBs with a sufficient degree of accuracy, and do not offer ranking of observations. The approach allows one to perform internal reliability analyses for linear (or linearized) systems with correlated and uncorrelated observations. In addition to the recommended reliability criteria, the less rigorous ones are also suggested. They can be applied in cases of unavoidable high correlation between the observations and for systems with small observational redundancy (e.g. levelling networks).

From this study it follows that different concepts of reliability measures can be evaluated with respect to the following requirements:

- range $\langle 0,1)$,

- possibility of ranking the observations,

- direct relation to MDB,

- geometric interpretation,

- possibility of creating interpretable criteria, and only to be used for correlated observations,

- possibility of disclosing abnormal responses of a system to gross errors.

For uncorrelated observations the redundancy numbers, used as reliability measures, satisfy all the four requirements. For correlated observations the reaction of a system to gross error is much more complex and some of the requirements are inconsistent. Studying the existing measures together with a proposed two-dimensional measure we can see that each of them satisfies only some of the items on this list. So the proposed approach should be considered as complementary to the existing approaches. 
Acknowledgments The research presented in this paper has been carried out within the Grant No. N N 526135134 founded by the National Research Council in Poland. The author is greatly indebted to this institution for their financial support. Special thanks go to one of the reviewers for his constructive comments and suggestions that enabled the author to further the analyses and consider the new approach in relation to the existing solutions.

Open Access This article is distributed under the terms of the Creative Commons Attribution Noncommercial License which permits any noncommercial use, distribution, and reproduction in any medium, provided the original author(s) and source are credited.

\section{References}

Baarda W (1968) A testing procedure for use in geodetic networks. Publications on Geodesy, New Series, vol 2. Netherlands Geodetic Commission, Delft, p 5

Chatterjee S, Hadi AS (1988) Sensitivity analysis in linear regression. Wiley, New York
Prószyński W (1994) Criteria for internal reliability of linear least squares models. Bull Géodésique 68:161-167

Prószyński W (1998) Reliability measures for the systems with correlated observations - a comparative study. In: Proceedings of the international symposium on Geodesy for geotechnical and structural engineering, Eisenstadt, Austria, pp. 216-221

Rao CR, Mitra SK (1971) Generalized inverse of matrices and its applications. Wiley, New York

Schaffrin B (1997) Reliability measures for correlated observations. J Eng Surv 123:126-137

Teunissen PJG (1990) Quality control in integrated navigation systems. IEEE Aerosp Electron Syst Mag 5(7):35-41

Teunissen PJG (1996) Testing theory, an introduction. Delft University Press, Delft

Teunissen PJG (1998) Minimal detectable biases of GPS data. J Geod $72: 236-244$

Van Mierlo J (1978) A testing procedure for analyzing geodetic deformation measurements. In: Proceedings of 2nd international symposium on deformation measurements, Bonn

Wang J, Chen Y (1994) On the reliability measure of observations. Acta Geodaet et Cartograph Sin. pp 42-51 (English edition) 